\title{
Searching for IMBHs in Galactic globular clusters through radial velocities of individual stars
}

\author{
Barbara Lanzoni \\ Department of Physics and Astronomy, University of Bologna, Viale Berti Pichat 6/2, 40127 \\ Bologna, Italy \\ email: barbara.lanzoni3@unibo.it
}

\begin{abstract}
I present an overview of our ongoing project aimed at building a new generation of velocity dispersion profiles ad rotation curves for a representative sample of Galactic globular clusters, from the the radial velocity of hundreds of individual stars distributed at different distances from the cluster center. The innermost portion of the profiles will be used to constrain the possible presence of intermediate-mass black holes. The adopted methodology consists of combining spectroscopic observations acquired with three different instruments at the ESO-VLT: the adaptive-optics assisted, integral field unit (IFU) spectrograph SINFONI for the innermost and highly crowded cluster cores, the multi-IFU spectrograph KMOS for the intermediate regions, and the multi-fiber instrument FLAMES/GIRAFFE-MEDUSA for the outskirts. The case of NGC 6388, representing the pilot project that motivated the entire program, is described in some details.
\end{abstract}

Keywords. globular clusters: general, globular clusters: individual (NGC 6388), stars: kinematics, techniques: spectroscopic, instrumentation: adaptive optics, black hole physics

\section{Introduction}

Confirming the existence of intermediate mass $\left(10^{3}-10^{4} M_{\odot}\right)$ black holes (IMBHs) would have a dramatic impact on a number of open astrophysical problems, ranging from the formation of supermassive BHs and their co-evolution with galaxies, to the origin of ultraluminous X-ray sources in nearby galaxies, up to the detection of gravitational waves (e.g. Gebhardt et al. 2005). However, the evidence gathered so far in support of the existence of IMBHs are inconclusive and controversial (see, e.g. Noyola et al. 2010, Anderson \& van der Marel 2010). Globular clusters (GCs) are thought to be the best places where to search for these elusive objects. In fact, the extrapolation of the "Magorrian relation" (Magorrian et al. 1998) down to the IMBH masses naturally leads to the GC mass regime. Moreover, numerical simulations have shown that the cores of dense star clusters are the ideal habitat for the formation of IMBHs (e.g. Portegies Zwart et al. 2004). For these reasons, the recent years have seen an increasing number of works dedicated to the search for IMBHs in GCs, exploiting all observational channels, ranging from the detection of X-ray and radio emission (see Strader et al. 2012 and Kirsten \& Vlemmings 2012, and references therein), to the detailed study of the shape of GC density and velocity dispersion profiles (e.g., Gebhardt et al. 2000; Gerssen et al. 2002, Lanzoni et al. 2007, Noyola et al. 2010, Lützgendorf et al. 2012).

In spite of such an effort, however, no firm conclusions could be drawn to date. This is mainly because of the great difficulties encountered from both the theoretical and the observational points of view. 


\section{Methodology}

To search for IMBHs in Galactic GCs (GGCs) we follow the "dynamical approach", i.e., we perform detailed studies of the cluster structure and dynamics with the goal of identifying the central density and velocity dispersion (VD) cusps theoretically predicted for stellar systems hosting an IMBH (e.g. Baumgardt, Makino, \& Hut 2005; Miocchi 2007).

Despite its importance, very little is empirically known to date about GGC internal dynamics, especially in the most crowded central regions. Recent results suggest that some insight can be obtained from the observations of exotic stellar populations, like blue straggler stars and millisecond pulsars (e.g., Ferraro et al. 2003, Ferraro et al. 2009a, Ferraro et al. 2009b, Ferraro et al. 2012). However, a detailed knowledge of the VD profile and rotation curve is still missing in the vast majority of GGCs because of observational difficulties. The velocity components on the plane of the sky can be obtained from internal proper motion measurements. However, this requires high-precision photometry and astrometry on quite long temporal baselines, together with precise estimates of GC distances. While this is starting to be feasible (thanks to the combination of multi-epoch HST observations and the improved techniques of data analysis; Anderson \& van der Marel 2010; Massari et al. 2013, Bellini et al. 2014), it is still very challenging in the high density central regions, where the crucial dynamical information constraining the presence of an IMBH is expected. The line of sight (los) velocity is in principle easier to obtain (through spectroscopy) and measurable in any cluster region and in GCs at any distance within the Galaxy. In practice, however, the standard approach commonly used in extra-Galactic astronomy (i.e. measuring the line broadening of integrated light spectra) is prone to a severe "shot noise bias": if a few bright stars are present, the acquired spectrum is completely dominated by their light and the line broadening is therefore not produced by the true VD of the underlying stellar population (e.g. Dubath, Meylan \& Mayor 1997). The alternative approach is to measure the dispersion about the mean of the radial velocities of statistically significant samples of individual stars. While this is safe from obvious biases, it is observationally challenging, especially in the highly crowded centers of GCs, requiring multi-object and spatially resolved spectroscopy.

Motivated by the astrophysical importance of determining the kinematical properties of GGCs, and because of the known shot noise bias affecting the integrated light spectroscopy method, we designed an ambitious project aimed at measuring the radial velocity of resolved stars along the entire radial extension of a representative sample of GGCs, by means of a multi-instrument approach: adaptive-optics (AO) assisted spectroscopy with SINFONI in the innermost, highly crowded regions, KMOS and FLAMES observations in the intermediate and external regions, respectively. Two large programmes are currently running at the Very Large Telescope of the European Southern Observatory (Prop. ID: 193.D-0232 and 195.D-0750, PI: F. R. Ferraro), collecting data for a representative sample of $\sim 30$ GGCs. Here we present the results obtained for the pilot project on NGC 6388 (see Lanzoni et al. 2013 and Lapenna et al. 2015).

\section{The pilot project: NGC 6388}

By using high spatial resolution $\left(0.025^{\prime \prime} \times 0.027^{\prime \prime} / \mathrm{pix}\right)$ data acquired with the HST /ACS High Resolution Channel (HRC), we discovered a shallow central density cusp in the inner $1^{\prime \prime}$ of this cluster and, from the comparison with King models including a central IMBH (Miocchi 2007), we concluded that a $\sim 6 \times 10^{3} M_{\odot} \mathrm{BH}$ could be hidden in NGC 6388 (Lanzoni et al. 2007). 


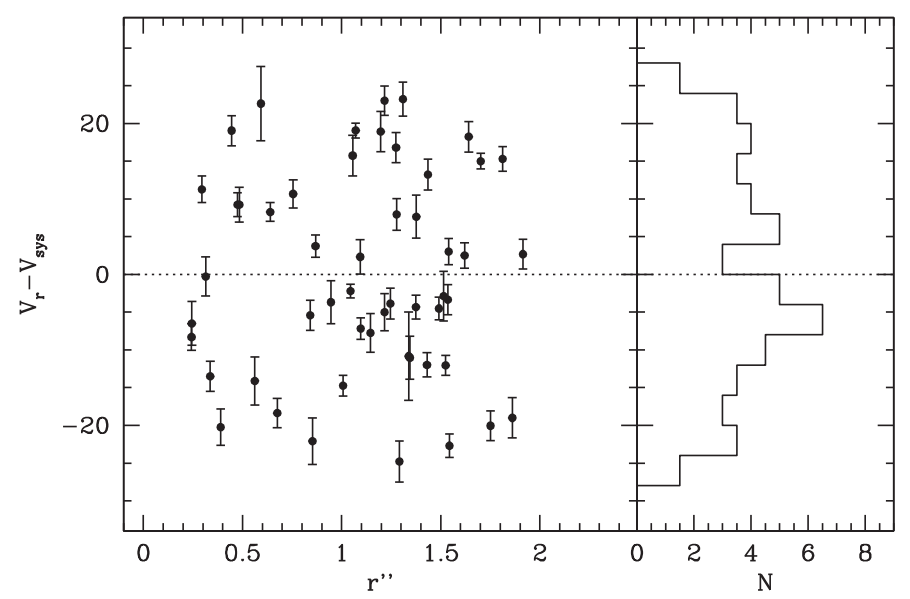

Figure 1. Left panel: distribution of the radial velocities measured for the 52 SINFONI targets in NGC 6388, referred to the cluster systemic velocity $V$ sys and plotted as function of the distance from the centre. Right panel: histogram of the radial velocity distribution. The apparent bimodality indicates the presence of systemic rotation.

To further investigate this possibility we then adopted the multi-instrument approach described above for determining the cluster VD profile and search for the central cusp expected in the presence of an IMBH.

SINFONI The innermost cluster regions have been properly investigated by exploiting the high spatial resolution capabilities of SINFONI (Eisenhauer et al. 2003), a near-IR (1.1-2.45 $\mu \mathrm{m})$ integral field spectrograph fed by an AO module and mounted on the YEPUN (VLT-UT4) telescope at the ESO Paranal Observatory. By using the 100 mas plate-scale and the $K$-band grating (sampling the $1.95-2.45 \mu \mathrm{m}$ wavelength range), we acquired spectra at a resolution $R=4000$ for $\sim 60$ stars located in a $3.2^{\prime \prime} \times 3.2^{\prime \prime}$ region centered on the cluster gravity center (as quoted in Lanzoni et al. 2007). From the ${ }^{12} \mathrm{C}^{16} \mathrm{O}$ band-heads (Origlia et al. 1997) and a few atomic lines we then measured the radial velocity $\left(V_{r}\right)$ of 52 giant stars located within $\sim 2^{\prime \prime}$ from the center of NGC 6388 .

KMOS KMOS is a second generation spectrograph equipped with 24 IFUs that can be allocated within a $7.2^{\prime}$ diameter field of view. Each IFU covers a projected area on the sky of about $2.8^{\prime \prime} \times 2.8^{\prime \prime}$, and it is sampled by an array of $14 \times 14$ spatial pixels (hereafter spaxels) with an angular size of $0.2^{\prime \prime}$ each. KMOS is equipped with four gratings providing a maximum spectral resolution $R$ between $\sim 3200$ and 4200 over the $0.8-2.5$ $\mu \mathrm{m}$ wavelength range. We have used the YJ grating and observed in the $1.00-1.35 \mu \mathrm{m}$ spectral range at a resolution $R \approx 3400$, corresponding to a sampling of about $1.75 \AA$ pixel $^{-1}$, i.e. $\sim 46 \mathrm{~km} \mathrm{~s}^{-1}$ pixel $^{-1}$ at $1.15 \mu \mathrm{m}$. By measuring several atomic lines for each star, we finally attained an average uncertainty of $\sim 3 \mathrm{~km} \mathrm{~s}^{-1}$ in the radial velocity estimates. With KMOS we thus measured $V_{r}$ for a total of 82 giant stars located within $\sim 70^{\prime \prime}$ from the center of NGC 6388 .

FLAMES The spectra of individual stars out to $\sim 600^{\prime \prime}$ have been acquired by using the ESO VLT multi-object spectrograph FLAMES, in the MEDUSA UVES+GIRAFFE combined mode. This provides 132 fibers to observe an equivalent number of targets in one single exposure, over a field of view of $25^{\prime}$ in diameter. Four pointings of $2320 \mathrm{~s}$ each have been obtained with the GIRAFFE grating HR21 (which samples the Ca II triplet spectral range at a resolution $R=16200$ ) and the UVES setup Red Arm 580, covering the wavelength range $4800 \AA<\lambda<6800 \AA$ at a resolution $R=47000$. The targets have been selected from the combined HST/ACS-WFC and ESO/WFI photometric catalog 


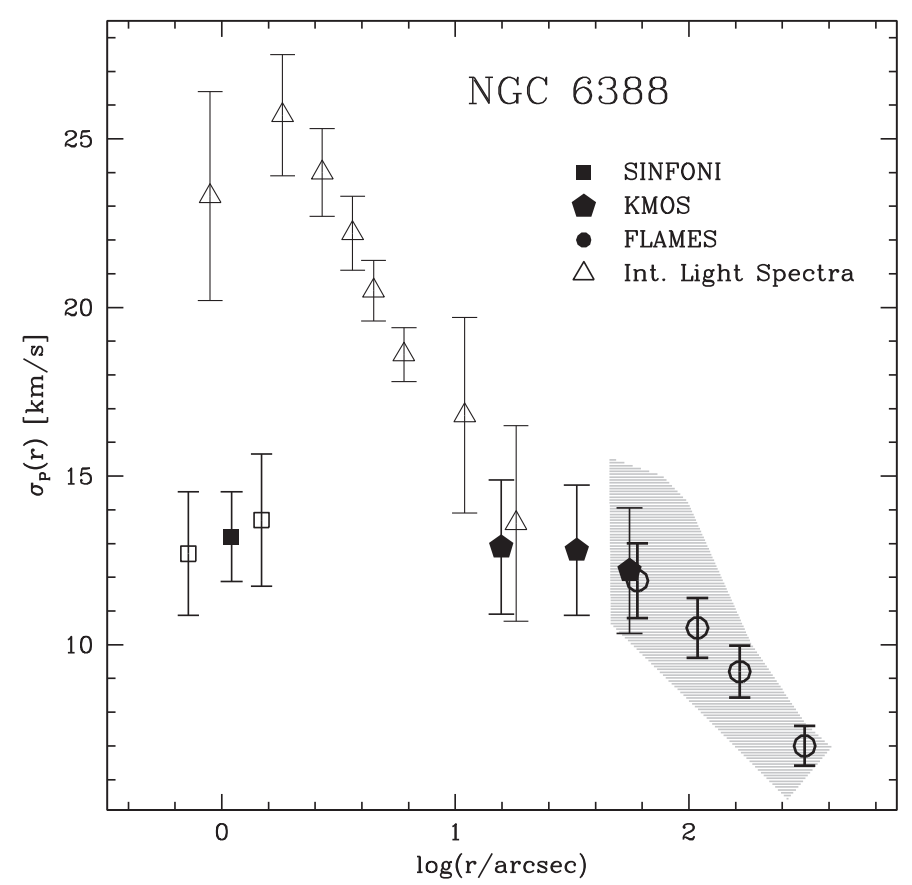

Figure 2. Line-of-sight velocity dispersion profile of NGC 6388 computed from the radial velocities of $\sim 400$ individual stars as measured with with SINFONI (squares), KMOS (pentagons), and FLAMES (empty circles). The the gray region indicates the dispersion obtained for different choices of the radial bins. The velocity dispersion profile obtained from integrated-light spectra (Lützgendorf et al. 2011) is also shown for comparison (empty triangles).

discussed in Lanzoni et al. (2007) and Dalessandro et al. (2008), considering only isolated objects (with no brighter stars within a circle of $1^{\prime \prime}$ radius), having $V<17$ and being located along the canonical evolutionary sequences of the color-magnitude diagram (CMD). By also including two additional data-sets retrieved from the ESO Archive, we finally obtained the radial velocities of about 270 member stars of the cluster.

In all cases, radial velocities have been measured by adopting the Fourier crosscorrelation method (Tonry et al. 1979) as implemented in the fxcor IRAF task. The observed spectrum is cross-correlated with a template of known radial velocity and a cross-correlation function (i.e., the probability of correlation as a function of the pixel shift) is computed. Then, this is fitted by using a Gaussian profile and its peak value is derived. Once the spectra are wavelength calibrated, the pixel shift obtained by the Gaussian fit is converted into radial velocity. We employed different reference template spectra according to the adopted spectral configuration and stellar type.

The systemic velocity of NGC 6388 has been computed by conservatively using all stars with radial velocities between 60 and $105 \mathrm{~km} \mathrm{~s}^{-1}$, deriving a value of $V_{r}=82.0 \pm 0.5$ $\mathrm{km} \mathrm{s}^{-1}$, in agreement with the literature (Harris 1996). Hereafter, $\widetilde{V}_{r}$ will indicate radial velocities referred to the cluster systemic velocity: $\widetilde{V}_{r} \equiv V_{r}-V_{\text {sys }}$.

Systemic rotation. The radial velocity distribution for the 52 SINFONI targets is plotted in Fig. 1. The histogram in the right-hand panel shows a clear bimodality, indicating the presence of systemic rotation. To more quantitatively investigate this possibility, we used the method fully described in Bellazzini et al. 2012. We considered a line passing through the cluster center with position angle PA varying between $0^{\circ}$ (North direction) and $90^{\circ}$ (East direction), by steps of $15^{\circ}$. For each value of PA, such a line splits the 


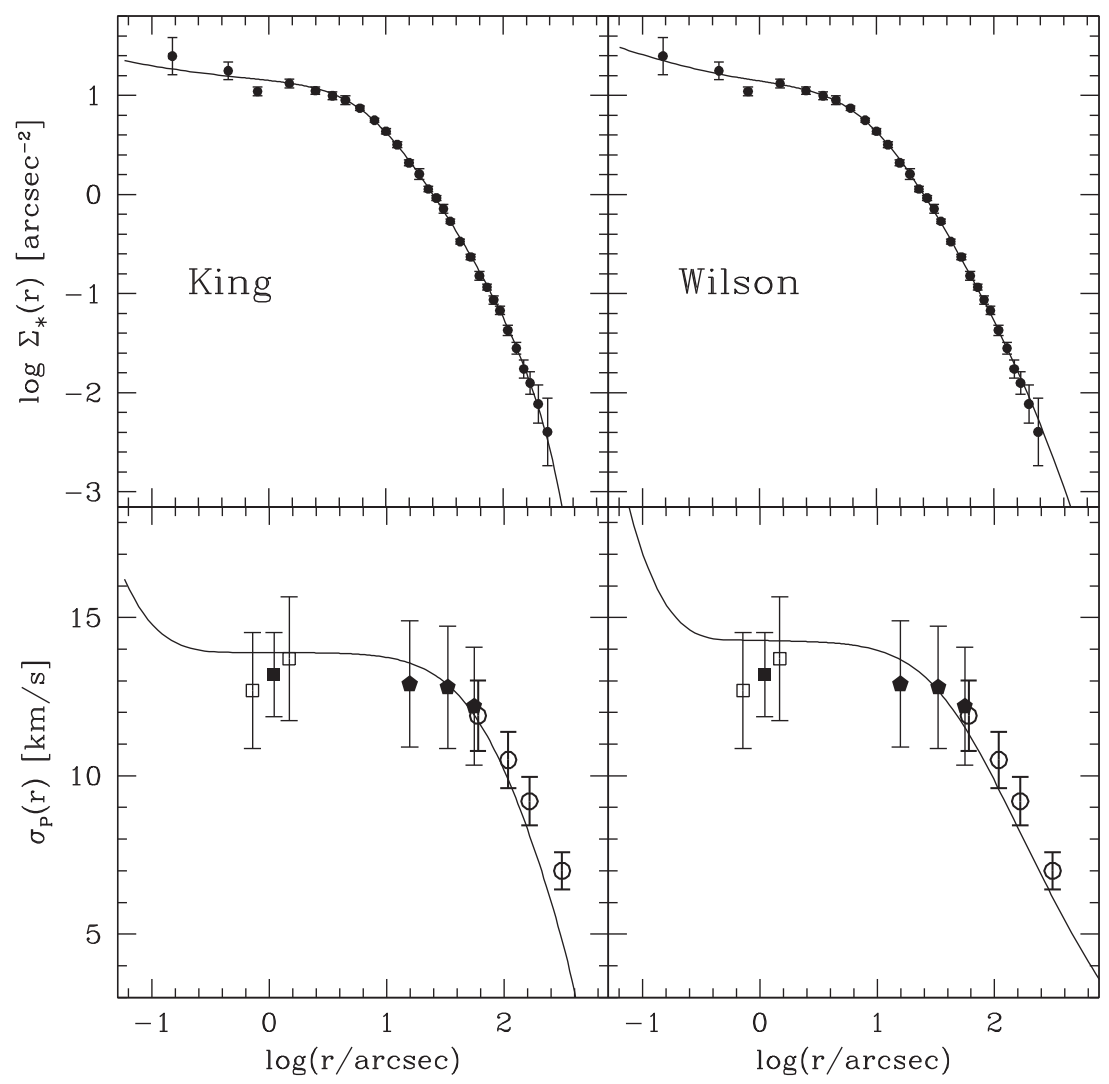

Figure 3. Comparison between the observations and the best-fit self-consistent King and Wilson models with central IMBH (left- and right-hand panels, respectively). The observed density profile (solid circles in the upper panels) is from Lanzoni et al. (2007). The observed velocity dispersion profiles are the same as in Fig. 2. An IMBH mass of $\sim 2000 M_{\odot}$ is assumed in these models.

SINFONI sample in two. The difference $\Delta\left\langle\widetilde{V}_{r}\right\rangle$ between the mean radial velocity of the two sub-samples was computed and has been found to show a coherent sinusoidal behavior, which is a signature of rotation. The best-fit position angle of the rotation axis is $\mathrm{PA}_{0}=11^{\circ}$ and the amplitude of the rotation curve $A_{\text {rot }}=8.5 \mathrm{~km} \mathrm{~s}^{-1}$. The KolmogorovSmirnov probability, however, indicates a not very high statistical significance, possibly due to the limited sample. Some hints of rotation are found also from the KMOS and FLAMES samples, but again the significance is weak. Additional data symmetrically sampling the cluster are needed before drawing any firm conclusion about ordered rotation in NGC 6388.

Velocity dispersion. To compute the projected VD profile, the surveyed area has been divided in a set of concentric annuli, chosen as a compromise between a good radial sampling and a statistically significant number $(>50)$ of stars. In each radial bin, $\sigma_{p}$ has been computed from the dispersion of the values of $\widetilde{V}_{r}$ measured for all the stars in the annulus, by following the Maximum Likelihood method described in Walker et al. (2006; see also Martin et al. 2007; Sollima et al. 2009). An iterative $3 \sigma$ clipping algorithm was applied in each bin. The error estimate is performed by following Pryor \& Meylan (1993). The resulting VD profile is shown in Figure 2. Given the number of stars in the SINFONI data set (52 objects in total) we computed $\sigma_{p}(r)$ by considering both one single 


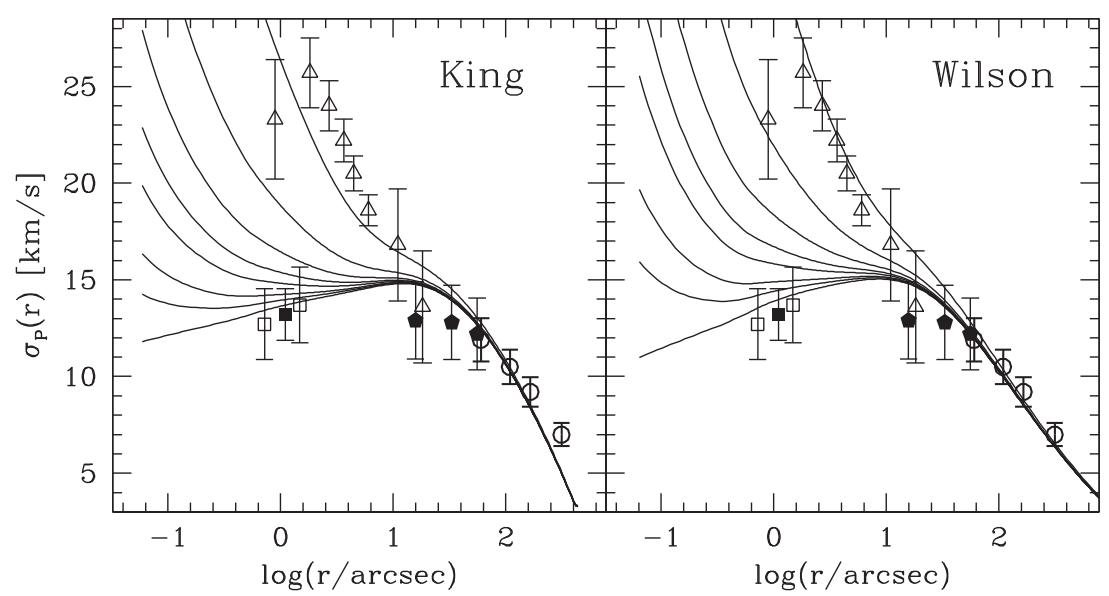

Figure 4. Comparison between the observed velocity dispersion profiles (the same as in Fig. 2) and two families of Jeans models. Left panel: solid lines correspond to Jeans models calculated from the King density profile shown in the upper-left panel of Fig. 3 and by assuming different black hole masses: from bottom to top, $M_{\mathrm{BH}}^{\mathrm{J}} / M_{\mathrm{BH}}^{\mathrm{sc}}=0,0.5,1,2,3,5,10,30$, with $M_{\mathrm{BH}}^{\mathrm{sc}}=2147 M_{\odot}$. Right panel: the same as in the left panel, but for Jeans models calculated from the Wilson density profile shown in the upper-right panel of Fig. 3. In this case, $M_{\mathrm{BH}}^{\mathrm{sc}}=2125 M_{\odot}$.

central bin (solid square in the figure) and two separate annuli (26 stars at $r<1.2^{\prime \prime}, 26$ stars beyond; empty squares). For the external data set we tried different sets of radial bins, finding consistent results (grey area in Fig. 2). The derived values are shown in the figure as black diamonds and empty circles for the KMOS and the FLAMES samples, respectively. We find that the velocity dispersion of NGC 6388 has a central $\left(r \sim 1^{\prime \prime}\right)$ value of $\sim 13 \mathrm{~km} \mathrm{~s}^{-1}$, stays approximately flat out to $r \sim 60^{\prime \prime}$, and then decreases to $\sim 7 \mathrm{~km} \mathrm{~s}^{-1}$ at $200^{\prime \prime}<r<600^{\prime \prime}$. If no $\sigma$ clipping algorithm is applied, the VD profile remains almost unchanged, with the only exception of the outermost data-point that rises to $\sim 8.9 \pm 0.8 \mathrm{~km} \mathrm{~s}^{-1}$.

The inner part $\left(r<10^{\prime \prime}\right)$ of the VD profile is clearly incompatible with the one obtained by Lützgendorf et al. (2011, hereafter L11) from the line broadening of integrated-light spectra, which shows a steep rise toward the cluster centre, up to values of $23-25 \mathrm{~km} \mathrm{~s}^{-1}$ (empty triangles in Fig. 2), suggesting the presence of an IMBH of $(1.7 \pm 0.9) \times 10^{4} M_{\odot}$. While the exact reason for such a disagreement is not completely clear, a detailed comparison between our radial velocity measurements and L11 radial velocity map suggests that their measure is affected by the shot noise bias mentioned above (see Sect. 4.1 and Fig. 12 in Lanzoni et al. 2013).

Comparison with models. In order to constraint the presence and the mass of a central IMBH in NGC 6388, we followed two different and complementary approaches. First, starting from a family of self-consistent models admitting a central IMBH, we selected the one that best reproduces the observed density and VD profiles. This provided us with the corresponding structural and kinematical parameters in physical units, including the IMBH mass. Second, we used the observed density profile and included a variable central point mass, to solve the spherical Jeans equation (this is what is done also in L11). We therefore obtained the corresponding family of VD profiles, which were then compared to the observations to constrain the $\mathrm{BH}$ mass. The first approach is more realistic in terms of the stellar mass-to-light ratio $(M / L)$, since it takes into account various populations of stars with different masses and different radial distributions (as it is indeed expected and observed in mass segregated GCs). Moreover it has the advantage that the models stem 
from a distribution function which is known a priori. Hence, the model consistency (i.e., the non-negativity of the distribution function in the phase-space) is under control and guaranteed by construction. However, the validity of the results is limited to the case in which the assumed models are the correct representation of the true structure and dynamics of the cluster. The second approach is more general, but we assume a constant $M / L$ ratio and the resulting models could be non-physical (e.g. Binney \& Tremaine 1987).

In all cases we assumed spherical symmetry and velocity isotropy. This seems to be reasonable for NGC 6388, which does not show significant ellipticity (Harris 1996) and is thought to be quite dynamically evolved (Ferraro et al. 2012; see also L11). In both approaches we considered King (1966) and Wilson (1975) distribution functions, which are known to well reproduce the observed density profiles of GGCs (e.g., McLaughlin \& van der Marel 2005; Miocchi et al. 2013).

As shown in Figures 3 and 4, our observed velocity dispersion profile is consistent with both the absence of a central IMBH, and the presence of a $\mathrm{BH}$ with a mass up to $\sim 2000 M_{\odot}$ (the latter possibility is mainly supported by the cusp in the density profile). The absence of a central IMBH in NGC 6388 is consistent with the results obtained from both X-ray and radio observation, which put an upper limit of $\sim 600 M_{\odot}$ to a possible compact dark mass in this cluster (Nucita et al. 2008; Cseh et al. 2010; Bozzo et al. 2011).

\section{Summary}

We are using a multi-instrument approach (SINFONI+KMOS+FLAMES at the ESOVLT) to measure the radial velocities of hundred individual stars distributed along the entire extension of $\sim 30$ Galactic GCs. The shape of the innermost portion of the velocity dispersion profile (and density distribution) will be used to constrain the presence of IMBHs in these systems. The pilot project on NGC 6388 demonstrates that this is the right route to properly determine GGC internal dynamics and pursue the "dynamical approach" to the search for IMBHs. Our study shows that no IMBH (or a $2000 M_{\odot} \mathrm{BH}$, at most) is required to account for the observations in this cluster.

Most of the results discussed in this talk have been obtained within the project CosmicLab (PI: Ferraro, see http://www.cosmic-lab.eu), a 5-year project funded by the European European Research Council under the 2010 Advanced Grant call (contract ERC2010-AdG-267675). I warmly thank the other team members involved in this research: Francesco Ferraro, Elena Valenti, Alessio Mucciarelli, Livia Origlia, Emilio Lapenna, Emanuele Dalessandro, Michele Bellazzini, Paolo Miocchi, Davide Massari and Cristina Pallanca.

\section{References}

Anderson, J. \& van der Marel, R. P. 2010, ApJ, 710, 1032

Baumgardt H., Makino J., \& Hut P., 2005, ApJ, 620, 238

Bellini, A., Anderson, J., van der Marel, R. P., et al. 2014, ApJ, 797, 115

Binney, J. \& Tremaine, S. 1987, Princeton, NJ, Princeton University Press

Bellazzini, M., Bragaglia, A., Carretta, E., et al. 2012, A\& A, 538, A18

Bozzo, E., Ferrigno, C., Stevens, J., et al. 2011, A\&A, 535, L1

Cseh, D., Kaaret, P., Corbel, S., et al. 2010, MNRAS, 406, 1049

Dubath, P., Meylan, G., \& Mayor, M. 1997, A\&A, 324, 505

Eisenhauer, F. et al., 2003, SPIE, 4841, 1548

Ferraro, F. R., Possenti, A., Sabbi, E., et al. 2003, ApJ, 595, 179 
Ferraro, F. R., Beccari, G., Dalessandro, E., et al. 2009a, Nature, 462, 1028

Ferraro, F. R., Dalessandro, E., Mucciarelli, A., et al. 2009b, Nature, 462, 483

Ferraro, F. R. et al., 2012, Nature, 492, 393

Dalessandro, E., Lanzoni, B., Ferraro, F. R., et al. 2008, ApJ, 677, 1069

Gebhardt, K., Pryor, C., O'Connell, R. D., Williams, T. B., \& Hesser, J. E. 2000, AJ, 119, 1268

Gebhardt, K., Rich, R. M., \& Ho, L. C. 2005, ApJ, 634, 1093

Gerssen, J., van der Marel, R. P., Gebhardt, K., et al. 2002, AJ, 124, 3270

Harris, W. E. 1996, AJ, 112, 1487

King I. R., 1966, $A J, 71,64$

Kirsten, F. \& Vlemmings, W. H. T. 2012, A\&AA, 542, A44

Lanzoni, B., Dalessandro, E., Ferraro, F. R., et al. 2007, ApJ, 668, L139

Lanzoni, B., Mucciarelli, A., Origlia, L., et al. 2013, ApJ, 769, 107

Lapenna, E., Origlia, L., Mucciarelli, A., et al. 2015, ApJ, 798, 23

Lützgendorf, N., Kissler-Patig, M., Noyola, E., et al. 2011, A\&AA, 533, A36 (L11)

Lützgendorf, N., Kissler-Patig, M., Gebhardt, K., et al. 2012, A\&AA, 542, A129

Massari, D., Bellini, A., Ferraro, F. R., et al. 2013, ApJ, 779, 81

Magorrian, J., Tremaine, S., Richstone, D., et al. 1998, AJ, 115, 2285

Martin, N. F., Ibata, R. A., Chapman, S. C., Irwin, M., \& Lewis, G. F. 2007, MNRAS, 380, 281

McLaughlin, D. E. \& van der Marel, R. P. 2005, ApJS, 161, 304

Miocchi, P. 2007, MNRAS, 381, 103

Miocchi, P. et al., 2013, ApJ, 774, 151

Noyola, E., Gebhardt, K., Kissler-Patig, M., et al. 2010, ApJ, 719, L60

Nucita, A. A., de Paolis, F., Ingrosso, G., Carpano, S., \& Guainazzi, M. 2008, A\&A A, 478, 763

Origlia, L., Ferraro, F. R., Fusi Pecci, F., \& Oliva, E. 1997, A\&̊A, 321, 859

Portegies Zwart, S. F., Baumgardt, H., Hut, P., Makino, J., \& McMillan, S. L. W. 2004, Nature, 428,724

Pryor, C. \& Meylan, G. 1993, Structure and Dynamics of Globular Clusters, 50, 357

Sollima, A., Bellazzini, M., Smart, R. L., et al. 2009, MNRAS, 396, 2183

Strader, J., Chomiuk, L., Maccarone, T. J., et al. 2012, ApJ, 750, L27

Tonry, J. \& Davis, M., 1979, AJ, 84, 1511

Walker, M. G., Mateo, M., Olszewski, E. W., et al. 2006, AJ, 131, 2114

Wilson, C. P. $1975, A J, 80,175$ 